\title{
Prevalencia de las úlceras por presión en una residencia asistida de mayores
}

Prevalence of pressure ulcers in an assisted residence for the elderly

J.M. Díaz Pizarro DUE. Consejería Bienestar Social CLM.

A.S. García Sánchez

A. Núñez Ballesteros

R. Osorio Díaz

Correspondencia:

José Miguel Díaz Pizarro

Avda. Lagunas de Ruidera no $12,1^{\circ} \mathrm{D}$

13005-Ciudad Real

Tfno.: 661131283

E-mail: josemdue@yahoo.com 


\section{RESUMEN}

Se recogieron datos de 282 residentes distribuidos en las diferentes áreas asistenciales de una residencia asistida de mayores, con el objetivo de analizar la prevalencia de lesiones por presión y sus características, así como el estado funcional y psíquico de los usuarios para poder realizar un estudio descriptivo de su situación actual.

Se observó una prevalencia del $13,47 \%$ de UPP con una mayor presencia en talones y una media de edad de 82,93 años.

Además, se analizaron factores que aumentan el riesgo de aparición de UPP, con especial atención a la presencia de incontinencia, uso de suplementos nutricionales y a las medidas de prevención como cambios posturales y colchones antiescaras.

\section{PALABRAS CLAVE}

Úlceras por presión, prevalencia, centros sociosanitarios, prevención, Índice de Barthel, Índice de Norton.

\section{SUMMARY}

We made a survey and collected data from 283 people distributed in the different welfare areas of an assisted residence for the elderly in order to analyse the prevalence of pressure ulcers as well as their functional and psychic conditions of the users to develop a descriptive report of their current situations.

A prevalence percentage of 13,47 was found, the sores in their heels being the most spread ones and within people at the average age of 82,93.

Besides, there were analyzed factors that increase the risk of PUs appearance with special attention to the presence of incontinence, use of nutritional supplements and the prevention measures as position changes and no injure mattresses.

\section{KEY WORDS}

Pressure sores, prevalence, socio-sanitary centres, prevention measures, Barthel Index, Norton Index.

\section{INTRODUCCIÓN}

Nuestro estudio se centra en una residencia asistida, donde el usuario requiere una gran cantidad de cuidados debido a la situación de dependencia que padece.

Esto ha hecho que nos marquemos como objetivo de este trabajo la realización de un estudio de prevalencia de úlceras por presión.

A nivel fisiológico, las alteraciones que se producen en la piel del anciano, además de las diferentes alteraciones orgánicas, hacen que en los mayores se produzcan UPP con una frecuencia elevada.

Si a esto le añadimos que la población que integra este tipo de residencias presenta otros factores de riesgo propios, como la dependencia de las actividades de la vida diaria, restricción de la movilidad, incontinencia urinaria y/o fecal, deficiente nutrición e hidratación, etc., obtenemos una aparición de UPP más frecuente y con peor pronóstico que en otros grupos de población (1).

\section{MATERIAL Y MÉTODO}

Los procedimientos utilizados en los residentes han sido realizados sin perjudicar en ningún momento la salud o el bienestar de los mismos.

Se realiza un estudio descriptivo transversal en una fecha determinada (6 de abril de 2006) sobre el grupo de población que integra una residencia asistida de mayores.

La residencia está formada por una Unidad asistencial, con el mayor número de residentes, un módulo de Alzheimer, con residentes que presentan alteraciones de convivencia y llamada así porque la mayoría presenta esta patología, y una Unidad de cuidados especiales (U.C.E.) con residentes que necesitan mayores cuidados y atenciones.

El análisis estadístico se realiza a través del software $\operatorname{SPSS}^{\circledR}$ v.11 y Microsoft Excel $^{\circledR} 2003$, a partir de registros de la hoja de recogida de datos confeccionada, donde incluimos:
- Edad, sexo, fecha de ingreso y estancia en la residencia.

- Presencia de UPP al ingreso y en el momento del estudio; grado y localización según el Grupo Nacional para el Estudio y Asesoramiento en Úlceras por Presión (GNEAUP) (2) y antigüedad de la lesión.

- Riesgo de presentar UPP según la puntuación en la Escala de Norton modificada por el INSALUD en el momento de estudio (riesgo muy alto $<10$; riesgo alto $=10-12$; riesgo medio=13-14 y riesgo mínimo/no riesgo> 14) (3).

- Situación funcional según la puntuación en el Índice de Barthel sugerida por Shah (independiente $=100$; dependiente escaso $=91-99$; dependiente moderado $=61-20$; dependiente severo $=21-60$ y dependiente to$\mathrm{tal}=20-0)(4)$.

- Presencia de incontinencia urinaria y/o fecal, diabetes, uso de suplementos nutricionales, utilización de superficies especiales para el manejo de la presión (SEMP) y cambios posturales como medios de prevención, así como diagnóstico de deterioro cognitivo mediante el Mini-Mental Test de Folstein (5).

\section{RESULTADOS}

En el momento elegido para la realización del estudio contamos en nuestro centro residencial con un total de 282 ancianos, de los cuales 11 se encuentran en la Unidad de cuidados especiales (U.C.E.) y 34 en el módulo de Alzheimer.

Encontramos que el número de mujeres -182- supera al de hombres, que es de 100.

La edad media de nuestros residentes es de 82,93 años, con una desviación típica de 7,58, donde el mayor cuenta con 105 años y el más joven tiene 55 . En cuanto a la estancia media de dichos residentes, es de 41,68 meses (3 años y medio aproximadamente).

Podemos observar que 38 residentes (un 13,47\%) presentaban úlceras por presión en el momento del estudio. Dieciséis de los ancianos (el 5,67\%) pre- 


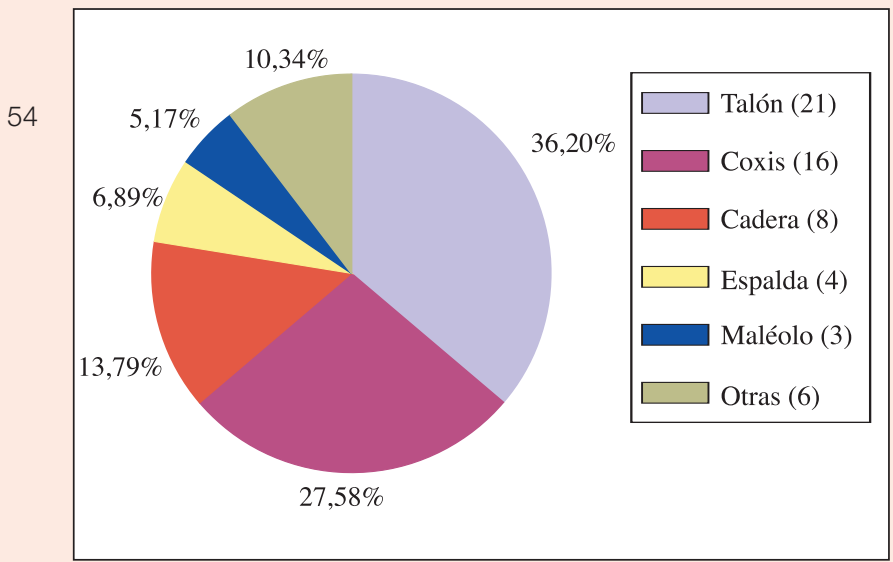

Fig. 1. Localizaciones de las UPP.

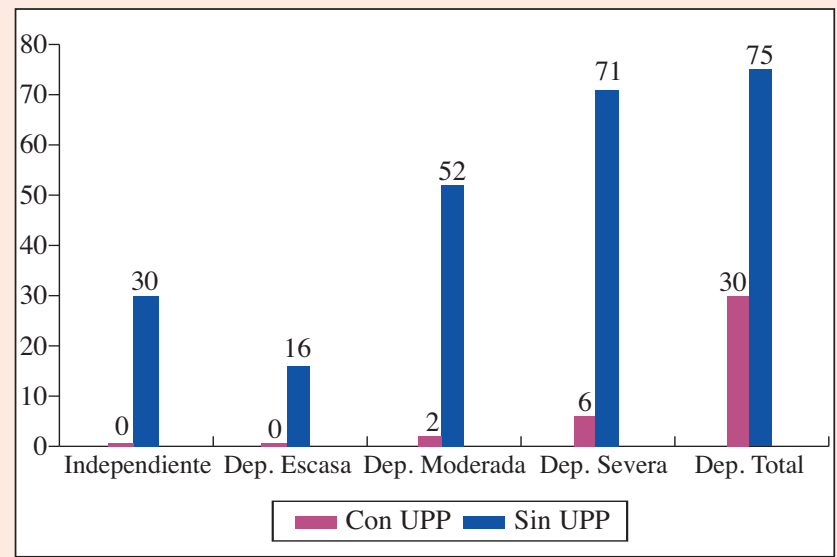

Fig. 2. Nivel de dependencia funcional de los residentes según padezcan o no úlcera por presión. sentaban UPP previa a su ingreso. En cuanto al tiempo medio de presencia de UPP, observamos que es de 3 meses y 22 días, con un tiempo máximo de presencia de un año y mínimo de 15 días.

De las 58 UPP halladas, su localización se distribuye según se indica en la Fig. 1.

En cuanto al grado de las UPP respecto del total de las mismas, se observa que 11 lesiones $(18,96 \%)$ corresponden a UPP grado I; $22(37,93 \%)$ son grado II; de grado III hay $19(32,75 \%)$ y 6 son de grado IV (10,34\%).

Del total de residentes que actualmente presentan alguna úlcera encontramos que 22 residentes (el $57,89 \%)$ solamente tienen una, 13 $(34,21 \%)$ cuentan con 2 , existen 2 residentes $(5,26 \%)$ con 3 UPP y sólo 1 $(2,63 \%)$ presenta 4 úlceras de forma simultánea.

Atendiendo a su distribución por las distintas Unidades, 6 de los residentes que se encuentran en la U.C.E. presentan UPP (lo que supone el 54,54\%). Mientras que eran 7 de los residentes de la Unidad de Alzheimer los que presentan el tipo de heridas del que nos ocupamos (el 20,58\%).

Si nos fijamos únicamente en el Índice de Barthel que presentan el total de residentes observamos que 30 (el $10,64 \%$ ) son funcionalmente independientes; 16 (el 5,67\%) presentan dependencia escasa; $54(19,15 \%)$ son moderadamente dependientes; 77 (27,30\%) tienen una dependencia severa y 105 presentan una dependencia total, constituyendo el $37,24 \%$ del total.
Si tomamos como medida la Escala de Norton modificada por el INSALUD cabe destacar que no existe riesgo de padecer UPP o el riesgo es mínimo para 129 residentes (el 45,74\%), mientras que 31 presentan riesgo medio (constituyendo el 10,99\%). Existen 59 residentes $(20,92 \%)$ que presentan riesgo alto y $63(22,34 \%)$ que presentan un riesgo muy alto de aparición de lesiones.

Se observa una media de estancia en el centro de 41,87 meses para los pacientes con UPP y de 41,67 meses para los que no presentan dichas lesiones.

En la Fig. 2 podemos ver el número de residentes que se encuadran en cada nivel de dependencia funcional, comparando a los que padecen úlcera por presión con los que no.

Del mismo modo, en la Fig. 3 vemos los residentes clasificados en función del riesgo de padecer UPP según la Escala de Norton, comparando a los que ya presentan dichas heridas con los que no.

En cuanto al empleo de suplementos nutricionales, éstos están siendo utilizados en 26 de los 38 residentes que tenían UPP (el 68,42\%), mientras que en los que no las presentan se observa cómo se utilizan estos suplementos en 66 de 244 residentes (27,04\%). En lo que respecta a la diabetes, hemos obtenido que de los residentes que padecían UPP, 10 son diabéticos $(26,31 \%)$; mientras que encontramos 79 residentes que sin padecer úlcera son diabéticos $(32,37 \%)$.
Encontramos que 29 de los 38 ancianos afectos de UPP presentan deterioro cognitivo, lo que supone un 76,31\%. Mientras que son 118 los que tienen dicho deterioro sin estar ulcerados (el 48,36\%).

De los 34 residentes que integran el módulo de Alzheimer, 32 presentan demencia (94,11\%); de los 11 que integran la U.C.E., la presentan 10 $(90,9 \%)$, y de los 237 ancianos distribuidos por las diferentes plantas, 105 tienen deterioro cognitivo (44,30\%).

Hemos observado que la prevalencia de incontinencia urinaria es del $100 \%$ para los 38 residentes que padecen úlcera y que existen 168 residentes con este tipo de incontinencia que mantienen la integridad de la piel $(68,85 \%)$. En cuanto a la incontinencia fecal, ésta es padecida por 32 de los ancianos con UPP $(84,21 \%)$ y por 105 de los que no presentan dichas lesiones (43,03\%).

Respecto al uso de SEMP, de los ancianos que presentan UPP, 28 utilizan estos dispositivos (73,68\%), mientras que en 31 de los residentes con la piel íntegra se adopta esta medida de prevención (12,70\%).

Además, se observa que 36 residentes presentan una dependencia severa o total y al mismo tiempo padecen UPP, 28 de ellos tienen SEMP $(77,77 \%)$. Para los 146 residentes que no tienen UPP pero se encuadran en ese mismo nivel de dependencia, encontramos que en 30 de ellos se utilizan dichos dispositivos (20,54\%).

Hemos encontrado que 33 residentes de los que tienen un riesgo alto o muy 


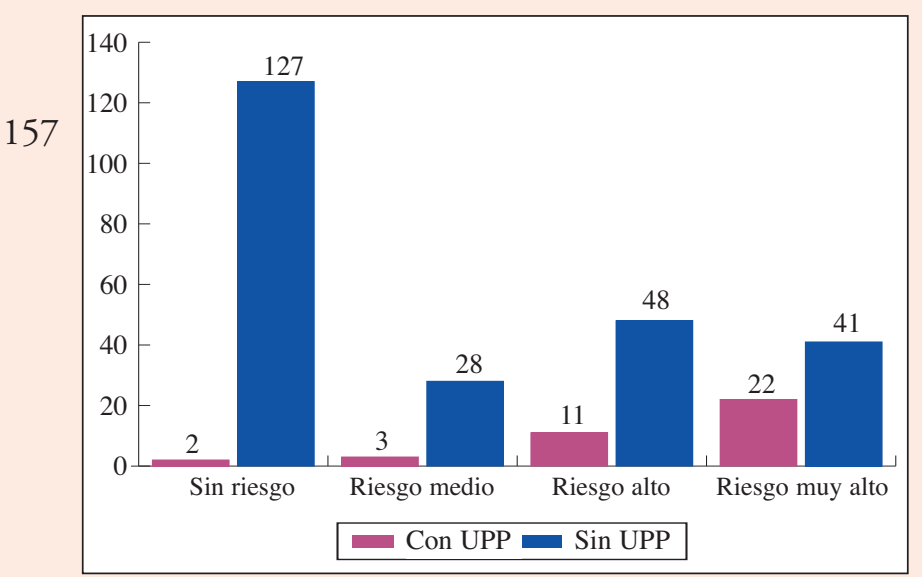

Fig. 3. Riesgo de padecer úlcera por presión de los residentes según padezcan o no esas lesiones actualmente.

alto de padecer UPP, la padecen actualmente; de ellos, en 26 se utilizan SEMP, es decir, en el $78,78 \%$. De igual modo existen 89 residentes con el mismo riesgo, pero sin presentar las lesiones actualmente; de este grupo, se están utilizando SEMP en 28 residentes (31,46\%).

Otra medida preventiva que hemos valorado en nuestro estudio es la realización de cambios posturales, obteniendo como resultado que se realizan en 35 de los residentes con alguna UPP (el $92,10 \%$ ) y en 138 de los 244 que no presentan ninguna $(56,55 \%)$.

\section{DISCUSIÓN}

Podemos ver cómo el mayor porcentaje de residentes ulcerados se encuentra localizado en la U.C.E., donde se pueden realizar un seguimiento más exhaustivo de la evolución de las
UPP y del propio residente.

Teniendo en cuenta que la población objeto del estudio pertenece a una residencia asistida, podemos comprobar cómo casi $2 / 3$ del total presentan una dependencia severa o total, según el Índice de Barthel utilizado.

Según los resultados obtenidos, los pacientes de la RAM con dependencia total, con deterioro cognitivo y con incontinencia urinaria y fecal tienen el mayor porcentaje de UPP.

Se observa que el porcentaje de residentes que presentaba úlceras por presión en el momento del estudio está por debajo de los estudios revisados, que cifran la prevalencia en centros sociosanitarios entre un 15 y $35 \%$ (6-9).

Existe un cambio en la localización de las UPP, apareciendo en primer lugar el talón, seguido del coxis, a diferencia de estudios anteriores, que clasificaban el coxis como principal zona de aparición de UPP, en parte por una mayor vigilancia en cambios posturales que previenen las heridas en coxis y trocánteres, pero no en talones (10-11).
No se observan diferencias con respecto a otros estudios en relación al número de úlceras que presentan los residentes ni en cuanto al tiempo de presencia de éstas (10-11).

El empleo de suplementos nutricionales tampoco es un dato a tener muy en cuenta, ya que algunos se instauran una vez aparecida la lesión y otros debido a indicaciones nutricionales, sin relación con la presencia o no de UPP (12).

En cuanto a la utilización de SEMP como medida de prevención de UPP, obtenemos que:

- Alrededor de un 75\% de los residentes con UPP usa estos dispositivos. En los residentes que no presentan lesiones se observa un uso escaso debido a una mayor utilización de éstos una vez aparecida la lesión.

- Teniendo como referencia el índice de Norton como principal medidor del riesgo de aparición de UPP, sería conveniente que el $100 \%$ de los residentes con un riesgo alto o muy alto utilizase SEMP, y no sólo el 78\% como indica el estudio, siendo deseable una mayor provisión de éstos.

\section{AGRADECIMIENTOS}

Queremos mostrar nuestro agradecimiento al centro por la oportunidad que nos ha brindado con la realización de este estudio y a todos los compañeros que han colaborado en la realización del mismo.

\section{BIBLIOGRAFÍA}

1. Torra i Bou JE, Rueda López J, Soldevilla Agreda JJ, Martínez Cuervo F, Verdú Soriano J. $1^{\text {er }}$ Estudio Nacional de Prevalencia de Úlceras por Presión en España. Epidemiología y variables definitorias de las lesiones y pacientes. Gerokomos 2006; 17: 154-72.

2. Grupo Nacional para el Estudio y Asesoramiento en Úlceras por Presión. Directrices generales para la prevención de las úlceras por presión. Castelldefels: GNEAUPP; 1996.

3. Elorriaga Ameyugo B, Royo Pascual D. Guía de cuidados enfermeros. Úlceras por presión. INSALUD. Madrid: Iberoamericana SA, 1996.

4. Shah S, Vanclay F, Cooper B. Improving the sensitivity of the Barthel Index for stroke rehabilitation. J Clin Epidemiol 1989; 42: 703-9.

5. Folstein M, Folstein S, McHugh P. Mini-Mental State. A practical method for grading the cognitive state of patients for the clinician. J Psych Res 1975; 12: 189-98.

6. Defloor T, Clark M, Witherow A, Colin D, Lindholm C, Moore Z. EPUAP statement on prevalence and incidence monitoring. 2005; 15 (3): 20-7.
7. Rodrigues I, Mégie MF. Prevalence of chronic wounds in Quebec home care: an exploratory study. Ostomy wound manage 2006; 52: 46-8, 50, 52-7.

8. Bours GJ, Halfens RJ, Abu-Saad HH, Grol, RT. Prevalence, prevention, and treatment of pressure ulcers: descriptive study in 89 institutions in the Netherlands. Res Nurs Health 2002; 25: 99-110.

9. Gunningberg L. Risk, prevalence and prevention of pressure ulcers in three Swedish healthcare settings. J Wound Care 2004; 13: 286-90.

10. Torra i Bou JE, Rueda López J, Soldevilla Agreda JJ, Martínez Cuervo F, Verdú Soriano J. $1^{\text {er }}$ Estudio Nacional de Prevalencia de Úlceras por Presión en España. Epidemiología y variables definitorias de las lesiones y pacientes. Gerokomos 2003; 14: 37-47.

11. Torra i Bou JE, Soldevilla Agreda JJ. Epidemiología de las Úlceras por Presión en España. Estudio piloto en la Comunidad Autónoma de La Rioja. Gerokomos 1999; 10: 75-87.

12. Hernández JC, Hernández F, Azira G. Prevalencia de la UPP en instituciones geriátricas. Enfermería Integral: Rev científica del Colegio Oficial de A.T.S de Valencia 2005; 70: 25-8.

\section{5}

